\title{
Hélice / tornillo: la denominación de un nuevo propulsor marino en el castellano del XIX
}

\author{
Cristina Villar Rey
}

Aunque la rosca de Arquímedes tiene más de dos mil años de antigüedad, su empleo en la propulsión de los buques es muy reciente. Los primeros ensayos comienzan a fines del siglo XVII, pero la aplicación verdaderamente práctica de la hélice a la navegación se debe a Francis Pettit Smith, un granjero inglés a quien le fue otorgada el 31 de mayo de 1836 una patente por un propulsor hélice de dos espiras completas. Casi simultáneamente, ese mismo año de 1836, hacía lo propio el ingeniero sueco Ericsson con un propulsor consistente en dos tambores en cuyas partes exteriores llevaban siete paletas helicoidales sujetas por un aro exterior; estaban las palas de una en opuesta inclinación de las de la otra y los tambores giraban en sentidos contrarios. Desde esa fecha los ensayos, patentes y pruebas se suceden hasta que la hélice consigue desterrar las ruedas de paletas al terreno de unos pocos usos muy concretos, convirtiéndose en el propulsor por antonomasia.

El conjunto de soluciones lingüísticas de que disponían los autores españoles para nombrar el nuevo referente era bastante amplio. Esta fase inicial de multiplicidad léxica fue resultado de las diferentes traducciones y de la adaptación de nuestro léxico a la terminología empleada en otras lenguas. En los autores del corpus examinado hemos podido recoger rosca, rosca hidráulica, rosca de Arquímedes, tornillo, tornillo propulsor, hélice, hélice submarino, hélice propulsor, superficie helizoide, rueda, propulsor submarino, propulsor helizoide, propulsor de hélice, helizoide, motor espiral. No obstante, antes de que triunfase el término que todavía hoy figura en el léxico de la construcción naval, la verdadera pugna se verificó entre éste y tornillo, elección que predomina en el uso de muchos de los autores que integran nuestro estudio; sin 
embargo, hacia 1860 la aceptación de la voz hélice era prácticamente unánime.

En un análisis sobre los mecanismos empleados para impulsar las embarcaciones, Tredgold ya cita la expresión rosca o tornillo de Arquímedes; se refiere a ella además con las denominaciones rosca hidráulica ó rosca de Arquímedes (1831: 405). En una única ocasión nombra al aparato propulsor con el término hélice, produciéndose de este modo una ampliación semántica de la voz, cuyo ámbito referencial se había limitado hasta entonces a la geometría, la arquitectura o la astronomía ${ }^{1}$ : "El agua habría adquirido ya toda la velocidad que la hélice podría comunicarla" (1831: 408).

Martínez Espinosa, por esos mismos años, en la entrada propelling screw de su diccionario, lo denomina hélice ó espiral, con la conveniente aclaración acerca de su finalidad debida a lo novedoso del mecanismo: "sustituye á las ruedas de paletas para dar movimiento á los buques de vapor" (1849: 335).

El término tornillo se utilizó en castellano durante los primeros años de introducción de este propulsor en la navegación. Aunque los principios teóricos matemáticos en los que se basa el funcionamiento de una hélice son los mismos que los de una rosca o tornillo, y los autores que intentaban explicar el funcionamiento de este aparato en sus tratados se ocupaban de desarrollarlos por extenso ${ }^{2}$, no consideramos que sea este

1 Son las tres acepciones del sustantivo hélice en la edición del Diccionario de la Academia de 1832. Es también posible documentar ejemplos del término con el significado de espira, es decir en su acepción perteneciente a la geometría, en las obras de Tredgold, Monjo, Carranza, etc., pues el nuevo propulsor tenía sus fundamentos teóricos en dicha forma geométrica de la que recibe una de sus denominaciones.

2 Así la presenta el Diccionario marítimo español de 1864 en la entrada correspondiente. Define en primer lugar el término en su acepción correspondiente a la Mecánica: "Curva en que se transforma una recta trazada en un plano, cuando este plano se arrolla en un cilindro. Si en tal disposicion se considera hueco el cilindro, y se imagina tirada una recta perpendicular al eje, desde un punto de la curva, se engendrará una superficie alabeada, llamada helicoide, haciendo que dicha recta se deslice por todos los puntos de la hélice, pero siempre en la misma disposicion respecto al eje del cilindro. El tornillo comun es la aplicación inmediata de esta superficie" (1864: 299). 
el motivo de esa denominación inicial. Se debería, principalmente, y una vez más, a la influencia del inglés, lengua en la que todavía en la actualidad, screw es la palabra normal para designar la hélice. Las obras inglesas y estadounidenses eran fuentes de consulta obligada para los marinos españoles, quienes al traducirlas o examinarlas mientras redactaban las suyas propias, vertían directamente al castellano el término inglés. Gustavo Fernández y Rodríguez, en su capítulo dedicado a los propulsores, confirma nuestra hipótesis: "Los primeros vapores de hélice en España se llamaban de tornillo, traduciendo sin duda esta palabra directamente de la inglesa screw" (1879: 295). No obstante, todos ellos emplean simultáneamente otras denominaciones, tal como comprobaremos de inmediato.

En 1850 Chacón aplica el término tornillo exclusivamente, aunque al comenzar su exposición acerca de dicho mecanismo titula el apartado "De la rosca ó tornillo como propulsor" (1858: 107); reserva hélice para aludir a la curva engendrada por la proyección de un filete sobre la superficie del cilindro que forma la rosca. Dos años más tarde convierte ambas voces en sinónimas, al emplearlas indistintamente para designar el mismo referente:

Ademas del exámen general de la máquina, se debe hacerlo muy particularmente de las ruedas dentadas que transmiten el movimiento á la hélice [...] Las articulaciones de las cigüeñas de los buques que carecen de ruedas dentadas requieren especial atencion, pues obrando directamente sobre el árbol del tornillo, sufren de lleno la reaccion que el agua ejerce sobre el tornillo en direccion de la quilla (1852: 107).

Inmediatamente, se presenta la acepción correspondiente a la navegación de vapor: "Hélice ó tornillo propulsor. Trozo del tornillo comun, de uno ó varios filetes muy elevados el cual girando rápidamente en el agua, encuentra en la inercia de esta una resistencia análoga a la que encontraría en una tuerca metálica; de donde resulta el movimiento progresivo ó la marcha del barco que lleva este mecanismo, y que tambien podria compararse con la de un bote, cuando se le hace caminar singlando con un remo por la popa" (1864: 299).

La definición que en 1869 por vez primera recogerá el Diccionario de la Academia para la voz hélice en su acepción naval, incide en este parentesco entre el aparato propulsor y el tornillo común (vid. infra). 
En 1859, al refundir y actualizar sus dos obras anteriores en un solo tomo, insiste en el empleo de tornillo de manera recurrente, aunque puntualiza que al "tornillo propulsor se le da tambien el nombre de hélice" (1859: 130). Cuando pocos años después revise y corrija la Cartilla de Roldán, para incluir en ella todas las novedades introducidas en la construcción naval desde la primera edición de 1831, sólo empleará la voz tornillo en una ocasión, acompañada de la forma que se estaba alzando con el predominio de la designación referencial: al tratar de las fuerzas motrices usadas para mover los buques habla de "la presion del vapor sobre un cuerpo de forma cualquiera que la traslada á unas ruedas de paletas ó á un tornillo ó hélice" (Roldán 1864: 18). En el resto de la obra hélice es la única elección.

En la misma década, la traducción del tratado de Mazaudier y Lombard mantiene el uso preferente de tornillo frente a hélice, aunque este segundo término se halla cuantitativamente más presente que en obras anteriores. Asimismo, es posible encontrar la lexía compuesta propulsor de hélice, precursora de lo que será la solución propulsor como término exclusivo para el referente hélice. Por otra parte, al tratar de los diversos sistemas que se estaban experimentando para desmontar dicho aparato en los buques con propulsión mixta de vapor y vela, recurre a un término del todo insólito para referirse a la hélice puesto que no se documenta en alusión a este referente en ninguna de las restantes obras consultadas. En una nota a pie de página, don José Barrera, el traductor, explica: "En la actualidad se logró el quitar dicha rueda y ponerla con mucha facilidad; construyendo esta de sólo dos paletas" (Mazaudier 1853: 193). Es fácil deducir que el uso de la voz rueda como sinónimo de hélice o tornillo es influencia de la denominación del propulsor más generalizado en la época, las ruedas de paletas.

Además, otra solución de los autores del Curso es superficie helicoide: "Para facilitar todavia mas el movimiento del agua á su salida del tornillo, y para hacer su construccion mas cómoda, se descompone la superficie helicóide en cierto número de aletas" (Mazaudier 1853: 191-192). En posteriores referencias al nuevo sistema de propulsión el traductor español lo designará "sistema de la helicóide" 
(1853: 195)³. Secundará la primera de estas propuestas léxicas Monjo i Pons:

\begin{abstract}
Algunos de estos defectos se han corregido ya, aplicando la misma fuerza motriz al movimiento jiratorio de la superficie helizoide o tornillo (1856: 19).

HELIZOIDE. La superficie alabeada descrita por el movimiento de una recta que, teniendo uno de sus estremos en el eje de un cilindro, $i$ formando con él un ángulo constante, va dando vueltas al primero, pasando por una hélice trazada sobre su superficie [...] Si dicho cuerpo está fijo, i conservándose siempre en contacto con la helizoide, jira el aparato, este se moverá lonjitudinalmente, hacia uno ú otro estremo según el sentido en que se verifique la rotacion. Véase TORNILLO, PROPULSOR (1856, Diccionario: 93) 4
\end{abstract}

Uno de los panoramas más ricos desde un punto de vista léxico nos lo proporciona la obra de don José de Carranza. Mientras que en el primer apartado dedicado a este propulsor dentro del capítulo que consagra a los métodos para impulsar los buques, leemos "Configuracion y descripcion del tornillo propulsor ó hélice" (1857: 92), en todos los restantes encabezamientos y en el $90 \%$ de las referencias al aparato en el cuerpo del texto, es tornillo el término escogidos: "Largo del tornillo", "Ángulo del tornillo", "Paso del tornillo", "Resbalamiento positivo y negativo del tornillo" (1857: 94), "Naturaleza y leyes del resbalamiento del tornillo"(1857: 97), “Accion centrífuga del tornillo" (1857: 98),

3 En el original francés "système de propulseur hélicoïde" (Mazaudier 1848: 72).

4 La segunda parte del Curso metódico de arquitectura naval de Juan Monjo i Pons está constituida por un Diccionario de arquitectura naval con las equivalencias en inglés $i$ en francés, con una paginación independiente de la del cuerpo de la obra (a esta sección nos referimos abreviadamente como Diccionario en nuestras citas). En él se recogen 1737 acepciones correspondientes a construcción naval, mecánica, maniobra, y a otras materias directamente vinculadas con ellas, tales como la geometría o la física.

5 Otras denominaciones presentes en el Tratado son propulsor submarino (1853: 485), hélice submarino (1853: 93) y propulsor mecánico (1853: 563). La tercera de estas expresiones se emplea indudablemente a fin de establecer una oposición manifiesta frente a los propulsores tradicionales que aprovechaban las fuerzas "naturales", bien se tratase del viento, los animales o el propio hombre. 
"Impulso del tornillo", "Rozamiento del tornillo" (1857: 101), "Inmersion del tornillo" (1857: 102), "De las mejores proporciones del tornillo propulsor" (1857: 104), "Eficiencia comparativa de diferentes clases de tornillos" (1857: 107), etc.

Ni siquiera recurre Carranza, a fin de evitar la continua repetición del mismo término en un párrafo, al sinónimo que él mismo había identificado en su introducción al aparato; en consecuencia, es probable encontrarse enunciados similares al siguiente:

La forma de tornillo mas frecuentemente empleada en Inglaterra, es el de dos palas ó espiras. El paso del tornillo se hace generalmente igual á su diámetro, ó un poco mayor, y el largo del tornillo, igual á un sesto del paso. La superficie impulsadora del tornillo se mide por el area del círculo descrito por las palas, la cual se denomina area del disco del tornillo (1857: 93).

Esta situación, no obstante, varía notablemente en los apéndices finales de la obra, cuando no expone fundamentos teóricos ni explicaciones mecánicas, sino que detalla el funcionamiento práctico y los resultados obtenidos en las distintas pruebas realizadas por muy diferentes buques ingleses, franceses o estadounidenses. La variedad léxica es más amplia en estos diferentes apartados y es posible hallar ejemplos muy enriquecedores para nuestro estudio.

Esas páginas finales permiten nuevamente confirmar la influencia de las fuentes consultadas por Carranza en la redacción del Tratado; hélice tiende a ser dominante en su análisis de buques franceses o en aquellos párrafos tomados de obras como el Traité de l'Hélice Propulsive de Paris, cuyas referencias el mismo autor proporciona, en tanto que al aludir a buques americanos o ingleses o al citar investigaciones de autores anglosajones, la forma elegida acostumbra ser tornillo.

El hélice que produjo el mejor resultado en el paquete 'Napoleon' tenia el resbalamiento del 25 por 100, que casi es el del 'Rattler.' En esto ha padecido una equivocacion tal vez Mr. Isherwood, porque el tornillo que dio mayor andar al 'Rattler' solo tenia el 10 ' 42 por 100 de resbalamiento (1857: 457).

Los únicos que conocemos que merezcan confianza son los del 'Peli- 
can' bajo la direccion de Messrs. Bourgois y Moll ejecutados por órden del gobierno francés y uno de ellos es casi semejante á los hélices propuestos y colocados despues al 'san Jacinto' (1857: 458).

Las pruebas del 'Rattler' y 'Alecto', y del 'Niger' y 'Basilisk', son los esperimentos de mas importancia verificados en la Armada Británica, y las insertaremos con algunas adiciones á la publicacion de Mr. Bourne [...] El impulso del eje del tornillo se midió con el dinamómetro [...] Se le hicieron 15 pies mas largas las salidas de agua, para la colocacion del tornillo [...] El resbalamiento del tornillo fue del 10'2 por ciento [...] El impulso sobre el eje del tornillo era de 3 tons. [...] Cuanto mas se aproximen tanto mas eficiente será la operación propulsora del tornillo (1857: 440).

La más que probable formación anglosajona de Carranza, sin embargo, se trasluce en sus elecciones léxicas que tienden predominantemente a las formas influidas por la lengua inglesa ${ }^{6}$. No obstante, al final del Glosario incluye una nota muy interesante, en donde aclara el empleo de ciertas denominaciones que, según deducimos de sus observaciones y de los testimonios proporcionados por las obras de la época, eran utilizadas de un modo confuso y, a juicio del autor, erróneamente:

Llamamos el tornillo ó el hélice al aparato propulsor, colocado en los delgados de la popa del buque; y la espira ó la hélice á la línea que con un paso ó avance dado se imagina enrollada sobre el propulsor, [...] el hélice ó helicoide se compondrá de tantas hélices como palas propulsoras tenga, si es de paso uniforme ó constante (1857: 33).

En efecto, el género de hélice servía para discriminar entre dos realidades distintas y en el texto de su obra Carranza diferencia claramente entre el masculino y el femenino y sus acepciones respectivas.

6 Aunque las formas inglesas eran screw propeller o propelling screw, y paralelamente en francés, hélice propulsive, observamos que Carranza y otros autores, si bien en algunos casos las traducen literalmente (de modo que leemos tornillo propulsor, hélice propulsor, etc.), en un momento inicial acostumbran reducirlas al primero de sus componentes que llegaría a convertirse en el término acostumbrado para designar el moderno propulsor. Más tarde, por un proceso similar, será el segundo constituyente de la lexía el que pasará a designar el mecanismo y propulsor se convertirá en sinónimo de hélice. Es un procedimiento semejante al que había provocado que las ruedas de paletas, por economía se designasen ruedas o sencillamente paletas. 
Sin embargo, la distinción no debía de estar tan clara para Barrera y Ariño, traductor del Curso de Mazaudier y Lombard, para quien hélice siempre es femenino, como lo era en la lengua de la que traducía, y así: "Se ha querido, por ejemplo, colocar dos hélices, una de cada lado", "una vez establecida la hélice" (1853: 193), "La razon del paso al diámetro de la hélice es mas considerable" (1853: 195), "Estando la hélice siempre sumergida" (1853: 197), "Perjudica la hélice el andar", "poner la hélice en su sitio" (1853: 198).

Sin embargo, sí descubrimos un único caso de probable vacilación en el género de hélice designando al aparato propulsor; en un mismo párrafo podemos leer:

Los hélices de bronce no pueden emplearse en los buques de hierro.

Finalmente es de desear, poder cuando se quiera, quitar la hélice ó volver á ponerla (1853: 193)

El hecho de que sea el único ejemplo de empleo en masculino en la totalidad del texto y de que se trate de una forma en plural, nos lleva a pensar que se deba a la correspondiente forma francesa, "les hélices en bronze" (1848: 68), que no iba acompañada de un adjetivo indicador de su género. Por tanto, una traducción un tanto apresurada, y la influencia del uso masculino que también era habitual en esos años, llevarían a don José Barrera a tal pequeño desliz.

Durante gran parte del siglo la forma masculina es la preferida para el aparato propulsor por los autores de manuales, artículos en publicaciones periódicas etc. Hasta 1860 Monjo es la única excepción, pero a partir de ese año las apariciones del femenino son cada vez más frecuentes, y así en los artículos de la Revista general de marina la mayoría de los colaboradores se inclinan por este género y son los menos quienes mantienen el masculino tradicional'.

En su afán por no traducir ni una sola página de obras extranjeras don Juan Monjo i Pons se muestra continuamente vacilante a la hora de

7 En 1877 Molinas todavía optará por el hélice si bien en el mismo año Fernández y Rodríguez designa el propulsor exclusivamente con el sustantivo femenino. 
dar nombre al nuevo aparato. Ofrece hasta seis soluciones diferentes sin inclinarse claramente por ninguna de ellas; son más frecuentes las expresiones compuestas por propulsor más un segundo término o un sintagma preposicional de carácter especificativo en relación, bien con la forma o emplazamiento, bien con los elementos caracterizadores del aparato:

Asi pues la invencion del propulsor submarino, debe considerarse como una adquisicion de la mayor trascendencia. Sin embargo no es probable que el tornillo llegue a desterrar enteramente á las ruedas, por que el mayor calado que requiere el primer mecanismo, le hace inadmisible para navegar en pocas aguas (1856: 192).

Aleta de propulsor. Cualquiera de las planchas helizoides que componen el tornillo o propulsor submarino (1856, Diccionario: 13$)$.

La barra cilíndrica de hierro colocada horizontalmente, que recibe la fuerza de las máquinas, i la transmite á dos ruedas laterales, ó á un propulsor de aletas sumerjido á popa. En los vapores de ruedas se coloca de babor á estribor [...] i en los de hélice se coloca de popa á proa debajo del nivel del agua (1856, Diccionario: 69).

PROPULSOR HELIZOIDE Ó SUBMARINO. Aparato de hierro ó de bronce colocado á popa i sumerjido en el agua, compuesto de un tubo metido en el estremo esterior del eje del vapor de hélice con dos ó mas aletas de forma helizoide (1856, Diccionario: 129).

En lo referente a la incorporación de la forma hélice a las obras lexicográficas, se produce cronológicamente en primer lugar en el diccionario de Chao de 1853 con la siguiente definición:

Pieza compuesta de un paso de rosca de dos o tres filos, que colocada a la parte de popa sustituye a las ruedas de palas en los modernos barcos de vapor.

Posteriormente, como hemos tenido ocasión de comprobar (vid. supra, nota 2) será recogida por el Diccionario marítimo español en $1864^{8}$, y el diccionario académico no la admitirá como término de marina sino hasta la edición de 1869 con esta definición:

8 Con respecto a la inclusión y el empleo en esta obra tanto de hélice como de tornillo, 
Trozo de rosca ó tornillo que se coloca á popa del buque de vapor y debajo del agua junto al timón. Se compone de dos ó más alas grandes, que giran alrededor de un eje, y encontrando en la inercia del agua la resistencia que ofrecería una tuerca, da impulso al buque (RAE 1869: s. v.).

Durante el último cuarto de siglo el uso de hélice ya se había afianzado. Autores como Fernández y Rodríguez, o Molinas son unánimes en la elección del término a pesar de que algunos creen necesarias ciertas aclaraciones con respecto al aparato y se refieren a él como "hélice o propulsor giratorio" (Garin 1895: 120) o bien aún ponen objeciones a la solución léxica adoptada, como Clairac:

Nombre dado, no muy propiamente, al trozo de tornillo comun, compuesto de uno ó varios filetes muy extendidos, ó aletas, que llevan sumergido á popa los buques de vapor, y le sirven de propulsor; pues girando rápidamente en el agua, encuentran en la inercia de ésta una resistencia análoga á la que encontraría en una tuerca; de donde resulta el movimiento progresivo ó la marcha del barco que lleva este mecanismo. También se llama tornillo y tornillo propulsor (1884, III: $532)^{9}$.

hay que constatar varios hechos. Dedica un extenso artículo a las hélices en donde proporciona una muy valiosa información sobre distintos tipos del propulsor y sus diferentes partes constitutivas; en la entrada que le corresponde aparece el término hélice como sinónimo de tornillo: "Hélice ó tornillo propulsor" (Lorenzo 1864: 299). Por otra parte, tornillo constituye una entrada independiente, cuya segunda acepción reza: "Nombre que muchos dan á la hélice" (Lorenzo 1864: 508). Paradójicamente, en los distintos artículos del Diccionario, sus autores prefieren la segunda de estas soluciones lingüísticas aunque las entradas correspondientes a ellos incluyan el término héli$c e$. De este modo, bajo "Chumacera de la hélice" encontramos "Recibe directamente el empuje del tornillo comunicándoselo al buque" (Lorenzo 1864: 198), y en conectar la hélice "unir el eje especial del tornillo, ó sea el que se halla entre los dos codastes, con el árbol ó eje" (Lorenzo 1864: 162). Cabe suponer como la causa de tales elecciones una cuestión de estilo, es decir, los redactores procurarían evitar la repetición del término. Sin embargo, no sería este el caso en las restantes y continuas apariciones de tornillo a lo largo del texto que revelan cierta predilección por el uso de dicha forma.

9 Este artículo se encuadra en el proyecto de investigación "La innovación léxica en el español del s. XIX" (XUGA10406A93). 


\section{BIBLIOGRAFÍA}

CARRANZA Y ECHEVERRÍA, José de (1857): Tratado de las máquinas de vapor aplicadas á la propulsion de los buques. Impreso por Real Orden para uso de los oficiales y Guardias Marinas de la Armada por el teniente de navio Don José de Carranza y Echeverría, Madrid, Imprenta de J. Martín Alegría.

CHACÓN Y ORTA, Francisco (1850): Breve idea de las máquinas de vapor y de sus aplicaciones á la navegacion. Redactada por D. Francisco Chacón y Orta, teniente de navio de la Armada de orden de S.M. se imprime por el Colegio Naval Militar, para uso de los alumnos de este establecimiento, Cádiz, Imprenta, librería y litografía de la Revista Médica [1859, $2^{\mathrm{a}}$ edic.].

(1852): Manejo de las máquinas de vapor de á bordo. Impreso de órden de S.M. para uso de los alumnos del Colegio Naval Militar, San Fernando, Imprenta y Librería Española a cargo de D. Juan Álvarez.

Снао, Eduardo, dir. (1853): Diccionario enciclopédico de la lengua espanola, con todas las vozes, frases, refranes y locuciones usadas en España y las Américas españolas, en el lenguajes comun antiguo y moderno; las de ciencias, artes y oficios; las notables de historia, biografía, mitolojía y geografía universal, y todas las particulares de las provincias españolas y americanas, por una sociedad de personas especiales en las letras, las ciencias y las artes, Madrid, Imprenta y librería de Gaspar y Roig, 2 vols.

ClAIRAC Y SÁENZ, Pelayo (1877-1891): Diccionario General de Arquitectura é Ingenieria, que comprende todas las voces y locuciones castellanas, tanto antiguas como modernas, usadas en las diversas artes de la construccion, con sus etimologías, citas de autoridades, historia, datos prácticos y equivalencias en francés, inglés é italiano, por D.Pelayo Clairac y Saenz, con una introducción por el Excmo. Sr. D. Eduardo Saavedra, Madrid, Talleres de impresion y reproduccion Zaragozano y Jayme, 5 vols.

FERNÁNDEZ Y RODRÍGUEZ, Gustavo (1877): Lecciones de construccion naval escritas para uso de los aspirantes á guardias marinas por Gustavo Fernández y Rodríguez, Madrid, Imprenta de T. Fortanet [1892, $2^{a}$ edición aumentada].

- (1879): Curso de máquinas de vapor que comprende la descripcion, el manejo y el entretenimiento de los principales tipos de calderas, 
máquinas y propulsores, usados en la navegacion de vapor, por Gustavo Fernández y Rodríguez, Madrid, Imprenta de Fortanet [1883, $2^{\mathrm{a}}$ ed.; 1891, $3^{\mathrm{a}}$ ed.].

GARIN Y SOCIATS, Arturo (1895): Nuevas teorías sobre formas, propulsión y aptitudes giratorias de los buques por Arturo Garin y Sociats, capitán de navio, Bilbao, Imprenta y encuadernación de Lucena é Hijos.

Lorenzo, José de, Gonzalo Murga y Martín Ferreiro (1864): Diccionario marítimo español: que ademas de las voces de navegacion y maniobra en los buques de vela contiene las equivalencias en frances, ingles é italiano y las mas usadas en los buques de vapor, formado con presencia de los mejores datos publicados hasta el dia, por José de Lorenzo, Gonzalo Murga y Martin Ferreiro, Madrid, Establecimiento tipográfico de T. Fortanet.

MARTíneZ DE EsPinOSA Y TACón, Juan José (1849): Diccionario marino español-inglés para el uso del Colegio Naval, Madrid, Imprenta de J. Martín Alegría.

MAZAUDIER, M. y M. LOMBARD (1848): Guide pratique pour la construction des bateaux à vapeur, à roues, à hélice et en fer, formant le complément du guide d'architecture navale ou exposé suivis dans les chantiers de la marine marchande et militaire, soit pour la construction des bateaux a vapeur a roues, a hélice et soit enfin pour la construction des bateaux a vapeur en fer, Paris, s. e.

- (1853): Curso completo y tratado práctico de arquitectura naval ó esposicion de los principios necesarios para la construccion de buques de guerra y mercantes; asi de vela, como vapores de madera y de hierro, con motor de ruedas ó hélice. Obra escrita en frances por M. Mazaudier y M. Lombard y vertida al castellano por el ingeniero de la armada D. José Barrera y Ariño, Ferrol, Imprenta y librería de Ricardo Pita.

Molinas Soler, Juan A. (1877): El maquinista naval. Obra dedicada al estudio de los conocimientos teórico-prácticos que hoy exige el gobierno para adquirir este título por Juan A. Molinas y Soler, ingeniero mecánico, Barcelona, Tipografía de Damián Vilarnau.

MONJO I PONS, Juan (1856): Curso metódico de arquitectura naval aplicada á la construccion de los buques mercantes. Obra compuesta en vista de las estrangeras mas modernas que tratan de la materia, en dos partes acompañadas de un ATLAS, é ilustradas con numerosas 
figuras intercaladas, por Juan Monjo i Pons, Barcelona, Imprenta de José Tauló.

Real ACADEMIa EsPañola (18327): Diccionario de la lengua castellana, Madrid, Imprenta Real.

Real ACADEMIa ESPAÑola (1869"1): Diccionario de la lengua castellana, Madrid, Manuel Rivadeneyra.

Revista general de Marina (1876-1899): Madrid, Depósito Hidrográfico.

RoLDÁN, Miguel (1864): Cartilla de construccion y manejo de los buques para instruccion de los guardias marinas, escrita por el capitan de fragata de la Armada Don Miguel Roldán en 1831. Corregida en 1863 por el capitan de navio de la misma Armada Don Francisco Chacon y Orta, Cádiz, Imprenta de la Revista médica [2 $2^{\mathrm{a}}$ edic., 1877, Madrid, Imprenta de T. Fortanet].

TREDGOLD, Thomas (1831): Tratado de las máquinas de vapor, y de su aplicacion a la navegacion, minas, manufacturas etc. Contiene la historia de la invencion y mejoras sucesivas de estas maquinas, la esposicion de su teoria y de las proporciones mas adecuadas de sus diversas partes, acompañada de un gran numero de tablas sinopticas que comprenden los resultados mas utiles para la practica; escrito en inglés por el ingeniero civil Th. Tredgold, traducido al francés por F. N. Mellet, y de este idioma al castellano de orden del Rey N. S. a quien va dedicado por Don Gerónimo de la Escosura, Madrid, Imprenta de D. León Amarita. 